\title{
Invariant and semi-invariant probabilistic normed spaces
}

\author{
M.B. Ghaemi ${ }^{\text {a, }}$, B. Lafuerza-Guillén ${ }^{\text {b }}$, S. Saiedinezhad ${ }^{a}$ \\ tSchool of Mathematics, Iran University of Science and Technology. Normak. Tehran, Iran \\ b Departamento de Estadistica y Matemática Aplicada, Untvezsidad de Almeria, Almerio E-04120, Sprin
}

\section{A R T I C E I NFO}

Atricle bistory:

Accepted 23 November 2008

\begin{abstract}
A B S T R A C T
Probabilistic metric spaces were introduced by Karl Menger. Nlsina. Schweizer and Sklar gave a general definition of probabilistic normed space based on the definition of Menger [1]. We intruduce the concept of semi-invariance among the $\mathrm{PN}$ spaces. In this paper we will find a sufficient condition for some PN spaces to be semi-imvariant. We will show that PN spaces are normal spaces. Urysohn's lemma, and Tietze extension theorem for them are proved.

22008 Elsevier Ltcl. All rights reserved.
\end{abstract}

\section{Introduction and preliminaries}

Menger proposed transferring the probabilistic notions of quantum mechanics from physics to the underlying geometry, The theory of probabilistic normed spaces (briefly. PN spaces) is important as a generalization of deterministic results of linear normed spaces and also in the study of random operator equations. The PN spaces may also provide us the appropriate tools to study the geometry of nuclear physics and have important applications in quantum particle physics particularly in strings' theory and in $\mathcal{E}^{\mathrm{x}}$ theory which were studied by El Naschie [2,3].

PN spaces were first defined by Šerstnev in 1962 (see [12]). Their definition was generalized in [1]. We recall the definition of probabilistic space briefly as given in [1], together with the notation that will be needed (see [11]). We shall consider the space of all distance probability distribution functions (briefly, d.f.'s), namely the set of all left-continuous and nondecreasing functions from $\overline{\mathbb{P}}$ into 0,1 such that $F(0)=0$ and $F(+\infty)=1$; here as usual, $\bar{R}:=\mathbb{R} \cup\{-\infty,+\infty\}$. The spaces of these functions will be denoted by $\Delta^{\prime}$, while the subset $D^{\prime} \subseteq \Delta^{\prime}$ will denote the set of all proper distance d.f.'s, namely those for which $f^{-} F(+x)=1$. Here $\ell^{-} f(x)$ denotes the left limit of the function $f$ at the point $x, f\left(f(x):=\lim _{r-x} f(t)\right.$. The space $\Delta^{*}$ is partially ordered by the usual pointwise ordering of functions, i.e.. $F \leqslant G$ if and only if $F(x) \leqslant G(x)$ for all $x$ in $R[10]$, For any $a \geqslant 0$, $\varepsilon_{i}$ is the d.f. given by

$$
\varepsilon_{\mathrm{II}}= \begin{cases}0 & \text { if } x \leqslant a \\ 1 & \text { if } x>a\end{cases}
$$

The space $\Delta^{+}$can be metrized in several ways [11], but we shall here adopt the Sibley metric $d_{5}$ which is the metric denoted by $d_{L}$ in [11],viz, the Lvy metric as a modified by Sibley [13]. If $F, G$ are d.f.'s and $h$ is in $\mid 0,1[$, let $[F$. G: $h]$ denote the condition:

$$
G(x) \leqslant F(x+h)+h \text { for all } x \in] 0, \frac{1}{h}[\text {. }
$$

\footnotetext{
- Corresponding author.

E-mail addresses; mghaemiètustacir (M.B. Ghaemi), blafuerz@ual.es (B. Lafuerza-Guilten), ssaicdinczhadeyahoocom (S. Saicdinezhad)
} 
Then the Sibley metric $d_{s}$ is defined by

$$
d_{5}(F, G):=\inf \{h \in[0,1[\text { both }\{F, G: h \mid \text { and }\{G, F: h] \text { hold }\} \text {. }
$$

Lemma 1.1. see [11], Lemma $4.2 .21 f d_{5}(f, G)-h>0$, then both $[F, G ; h]$ and $\left.G, F ; h\right]$ hold.

Definition 1.2. A triangular norm or, briefly, a $t$-norm is a function $\left.T:(0,1)^{2} \rightarrow \mid 0,1\right]$ that satisfies the following conditions:

(T1) it is commutative, i.e., $T(s, t)=T(t, s)$ for all $s$ and $t$ in $[0,1]$ :

(T2) it is associative, i.e., $T(T(s, t), u)=T(s, T(t, u))$ for all $s, t$ and $u$ in $0,1 \mid$ :

(T3) it is increasing in each place, i.e., $T(s, t) \leqslant T\left(s^{\prime}, t\right)$ for all $t \in[0,1]$ whenever $s \leqslant s^{\prime}$ :

(T4) it satisfies the boundary condition $T(1, t)=t$ for every $t \in[0,1]$.

The most important $t$-norms are the minimum $M$, the product $n$, the Lukasiewicz $t$-norm $W$ and the drastic product $D$ given by

$$
\begin{aligned}
& M(x, y):=\min \{x, y\}, \\
& \Pi(x, y):=x y . \\
& W(x, y):=\max \{0, x+y-1\}, \\
& D(x, y):= \begin{cases}\min \{x, y\} & \text { if } \max \{x, y\}=1, \\
0 & \text { otherwise, }\end{cases}
\end{aligned}
$$

A triangle function is a binary operation on $\Delta^{\prime}$. namely a function $\tau: \Delta^{\prime} \times \Delta^{\prime}-\Delta^{\prime}$ that is associative, commutative, nondecreasing in each place and has $\varepsilon_{0}$ as identity, that is, for all $F, G$ and $H$ in $\Delta$ :

(TF1) $\tau(\tau(F, G), H)=\tau(F, \tau(G, H))$.

(TF2) $\tau(F, G)=\tau(G, F)$,

(TF3) $F \leqslant G \Rightarrow \tau(F, H) \leqslant \tau(G, H)$,

(TF4) $\tau\left(F, \varepsilon_{0}\right)=\tau\left(\varepsilon_{0}, F\right)=F$.

In particular, under the usual pointwise ordering of functions, $\varepsilon_{0}$ is the maximal element of $\Delta^{-}$. Moreover, a triangle function is contintous if it is continuous in the metric space $\left(\Delta . d_{5}\right)$.

Typical continuous triangle functions are

(a) $\tau_{r}(F, G)(x)=\sup _{s+r-x} T(F(s), G(t))$.

(b) $\tau_{\gamma} \cdot(F, G)(x)=$ inf $_{s+t-x} T^{*}(F(s), G(t))$.

(c) $\Pi_{\mathrm{T}}(F, G)(x)=T(F(x), G(x))$,

and here $T$ is a continuous $t$-norm.

The definition below is more general; it has been proposed in [1].

Definition 1.3. A Probabilistic normed space (briefly. PN space) is a quadruple $\left(V, v, \tau, \tau^{*}\right)$, where $V$ is a real vector space, $\tau$ and $\tau^{*}$ are continuous triangle functions with $\tau \leqslant \tau^{*}$ and $v^{\prime}$ is a mapping (the probabilistic norm) from $V$ into $\Delta^{+}$, such that for every choice of $p$ and $q$ in $V$ the following hold:

(N1) $v_{p}=\varepsilon_{1}$ if and only if. $p=a(B$ is the null vector in $V$ ):

(N2) $v_{-p}=v_{p}$;

(N3) $v_{p+4} \geqslant \tau\left(v_{p}, v_{q}\right):$

(N4) $v_{p} \leqslant \tau^{*}\left(v_{i}, v_{11} ; p\right)$ for every $i \in[0,1$.

There is a natural topology in a PN space $\left(V, v, \tau, \tau^{*}\right)$, called strong topology; it is defined for $p \in V$ and $t>0$, by the neighborhoods

$$
N_{p}(t):=\left\{q \in V ; V_{4}, t(t)>1-t\right\}=\left\{q \in V ; d_{S}\left\langle V_{Q, D}, \varepsilon_{0}\right\rangle<t\right\}
$$

We recall that a set $A$ in a $P N$ space $\left(V, v, \tau, \tau^{*}\right)$, is said to be $D$-bonded if its probabilistic radius $R_{A}$ belong to $D^{+}$, where

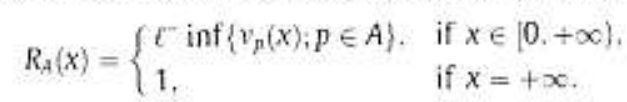

There are special PN spaces, only some of which we list below: for the others we refer to [5]. 
When there is a continuous $t$-norm $T$ (see [11]) such that $\tau=\tau_{T}$ and $\tau^{+}=\tau_{\mathrm{f}^{-}}$, where $T^{\prime}(x, y)=1-T(1-x, 1-y\rangle$,

$$
\tau_{T}\langle F, G)(x):=\sup _{s+t=x} T(F(s), G(t)) \text { and } \tau_{T}(F, G)(x):=\inf _{s+t-x} T^{*}(F(s), G(t))
$$

the PN space $\left(V, v, \tau_{\gamma}, \tau_{r}\right)$ is called a Menger PN space, and is denoted by $(V, V, T)$.

A PN space is called a Serstrtev space if it satisfies (N1). (N3) and the following condition, which implies both (N2) and (N4)

$$
\forall p \in V \quad \forall \alpha \in \mathbb{R} \backslash\{0\} \quad \forall x>0 \quad v_{r p}(x)=v_{p}\left(\frac{x}{x}\right) .
$$

One speaks of an equilateral PN space when there is $F \in \Delta^{+}$different from both $\epsilon_{0}$ and $\epsilon_{\alpha}$ such that, for every $p-\theta, v_{p}=F$. and when $\tau=\tau^{*}=\mathbf{M}$, which is the triangle function defined for $G$ and $H$ in $\Delta^{\prime}$ by $M(G, H)(x):=\min \langle G(x), H(x)\}$.

Let $G \in \Delta^{+}$be different frum $\epsilon_{0}$ and from $\epsilon_{\mathrm{x}}$ and let $(V,\|\cdot\|)$ be a normed space; then define, for $p \neq \theta$,

$$
v_{\mathrm{p}}(x):=G\left(\frac{x}{\|p\|}\right) .
$$

Then $(V . v . M)$ is a Menger space denoted by $(V .\|\cdot\|, G, M\rangle\langle M(x, y):=\min \{x, y\})$. In the same conditions, if $v$ is defined by

$$
v_{p}(x):=G\left(\frac{x}{\|p\|^{x}}\right) \text {. }
$$

with $\alpha \geqslant 0$, then the pair $(V, v)$ is a PSN space called $\alpha$-simple and it is denoted by $(V, \|-\{, G: \alpha)$. Let $(\Omega, \alpha, P)$ be a probability space, $(V,\|-\|)$ a normed space and $S$ a vector space of $V$-valued random variables (possibly, the entire space). For cvery $p \in S$ and for every $x \in \mathbf{R}$. let $v: S \rightarrow \Delta^{+}$be defined by $\left.v_{p}(x):=\rho(0)<\Omega:\|p(\alpha)\|<x\right\}$ : then $(S, v)$ is called an E-normed space (briefly. EN space) with base $(\Omega, ., P)$ and target $(V,\|\cdot\|)$. Every EN space $(S, v)$ is a PPN space under $\tau_{W}$ and $\tau_{M}$. It is said to be cononical if it is a PN space under the same two triangle functions. In this latter case, it is a Serstnev space. See $[5,8]$ for properties of PN spaces.

If $v_{p}(x)$ is viewed as the probability $P(\| p \mid<x)$ that the usual norm of $p$ is less than $x$, then, the fact that, for some $p \in V, v_{p}$ does not belong to $\mathscr{S}^{\prime}$ means that $\left.P(\mid \mathrm{p}) \mid<+\infty\right)<1$; this is to be regarded as being "odd". Therefore we shall call strict any PN space $\left(V, v, \tau, \tau^{*}\right)$ such that $v(V) \subseteq V^{+}$. or, equivalently, such that $v_{p}$ belongs to $\ell^{+}$for every $p \in V$.

Definition 1.4. A copula is a function $C \cdot[0,1]^{2}-[0,1]$ that satisfies the following conditions:

(C1) for every $t \in[0,1], C(0, t)=C(t, 0)=0$ and $C(1, t)=C(t, 1)=t$;

(C2) $C$ is 2 -increasing, i.e., for all $s, s^{\prime}, t$ and $t^{\prime}$ in $[0,1]$, with $s \leqslant s^{\prime}$ and $t \leqslant t^{\prime}$.

$$
C\left(s^{\prime}, t^{\prime}\right)-C\left(s^{\prime}, t\right)-C\left(s, t^{\prime}\right)+C(s, t) \geqslant 0
$$

It follows from the definition that every copula $C$ is increasing in each place.

Moreover for any copula $C$ one has $W \leqslant C \leqslant M$.

\section{Invariant and semi-invariant PN spaces}

In any PN space $\left(V, v, \tau, \tau^{+}\right)$with regard to the distance $d_{s,}$ one has, in general, the following:

$$
d_{5}\left(v_{0}-4, \varepsilon_{0}\right) \neq d_{5}\left(v_{p}, v_{q}\right) \text {. }
$$

But it would be interesting to know in which cases and under which conditions the inequality $d_{5}\left(v_{p} q-\varepsilon_{0}\right) \geqslant d_{5}\left(v_{p}, v_{q}\right\}$ holds. In this case, the corresponding relationship in functional analysis is

$$
\|p-q\| \geqslant \mid\|p\|-\|q\| \text {. }
$$

Definition 2.1. The probabilistic normed space $\left(V, V, \tau, \tau^{\prime}\right)$ is said to be invariant, if for every $p . q \in V$ we have $d_{s}\left(v_{y-q}, \varepsilon_{0}\right)=d_{s}\left(v_{p}, v_{4}\right)$.

Definition 2.2. The probabilistic normed space $\left\langle V, r, \tau, \tau^{*}\right\rangle$ is said to be semi-invariant, if for every $p . q \in V$ we have $d_{5}\left(v_{p-q}, \varepsilon_{0}\right) \geqslant d_{5}\left(v_{p}, v_{4}\right)$

Example 2.3. The quadruple $\left(\left[, v, \tau_{\pi}, \tau_{n}\right)\right.$ where $r: \& \rightarrow \Delta^{+}$is defined by

$$
v_{p}(x)= \begin{cases}0, & \text { if } x=0, \\ \exp (-\sqrt{\|p\|}), & \text { if } 0<x<+\infty \\ 1, & \text { if } x=+\infty\end{cases}
$$


and $v_{0}=\varepsilon_{0}$ is a semi-invariant PN space strictly.

Proof. $\left(R, v, \tau_{\pi}, \tau_{\pi}\right)$ is a PN space of Menger that is not a Sertnev space [1]. By theorem 4.3 .3 of [11] one has $d_{5}\left(v_{p}, \varepsilon_{0}\right\}=\inf \left\{h \in 0.1\left[v_{p-q}\left(h^{+}\right\}>1-h\right\}=\inf \left\{h \in \mid 0.1\left[: \exp \left(-\|p-q\|^{1 / 2}\right\}>1-h\right)=1-\exp \left(-\|p-q\|^{1 / 2}\right)\right.\right.$.

Now let us see $d_{s}\left(v_{p}, v_{q}\right)$. Suppose, without lost of generality. $\|p\| \geqslant\|q\|$. Then for every $h \in \mid 0,1[$ and for all $r>0$, particularly for $t \in[0,1 / h]$, one has

$$
v_{p}(t) \leqslant v_{q}(t+h)+h
$$

so that, in our example, the condition $\left[v_{q}, v_{p} ; h\right]$ clearly holds. The other condition $\left[v_{p}, v_{n} ; h\right]$ says that

$$
h \geqslant \exp \left(-\|q\|^{1 / 2}\right)-\exp \left(-\|p\|^{1 / 2}\right)
$$

Consequently, if $p \neq q$.

$$
d_{S}\left(v_{p}, v_{q}\right)=\exp \left(-(\min \{\|q\|,\|p\|\})^{1 / 2}\right)-\exp \left(-\left(\max \left\{\left\|\mathfrak{T}_{\|} \mid \cdot\right\| p \|\right\}\right)^{1 / 2}\right) .
$$

holds. And taking into account the following relations

$$
\begin{aligned}
\sqrt{\|p\|} & \leqslant \sqrt{\mid p-q\|+\| q \|} \leqslant \sqrt{\|p-q\|}+\sqrt{\|q\|} \\
& \Rightarrow \exp (-\sqrt{\|p\|}\rangle \geqslant \exp (-\sqrt{\|p-q\|}) \cdot \exp (-\sqrt{\|q\|}) \\
& \Rightarrow \exp (-\sqrt{\|q\|})-\exp (-\sqrt{\|p\|}) \leqslant \exp (-\sqrt{\mid q \|})-\exp (-\sqrt{\mid p-q \|}) \cdot \exp (-\sqrt{\|q\|}) \\
& =\exp (-\sqrt{\|q\|}) \cdot[1-\exp (-\sqrt{|p-q|}) \mid \leqslant 1-\exp (-\sqrt{\|p-q\|}) .
\end{aligned}
$$

one has finally the strict inequality

$$
d_{5}\left(v_{p-4}, \varepsilon_{0}\right)>d_{5}\left(v_{p}, v_{4}\right)
$$

Lemma 2.4. If $a, b \in[0,+\infty]$, then the statement

$$
d_{s}\left(\varepsilon_{a}, \varepsilon_{b}\right)=\min \left\{1 \cdot \frac{1}{\min \{a \cdot b\}},|a-b|\right\}
$$

holds. Particularly, the cases $b=0$ and $b=+\infty$ are

$$
\begin{aligned}
& d_{5}\left(\varepsilon_{i}, \varepsilon_{0}\right)=\min \{1, a\} \\
& d_{5}\left(\varepsilon_{x}, \varepsilon_{x}\right)=\min \{1,1 / a\}
\end{aligned}
$$

Proof. Based on the definition (1.1) one has

$$
d_{5}\left(\varepsilon_{0}, \varepsilon_{b}\right)=\inf \left(h \in[0,1] \text {; both }\left[\varepsilon_{a}, \varepsilon_{b}, h\right] \text { and }\left[\varepsilon_{b}, \varepsilon_{a}, h_{j} \text {, hold }\right\}\right. \text {. }
$$

Let us recall that for every $x \in j 0, h_{h}\left[\right.$ the relation $\left.\mid \varepsilon_{g}, \varepsilon_{b}, h\right] \Longleftrightarrow \varepsilon_{n}(x) \leqslant \varepsilon_{a}(x+h)+h$ holds.

Consequently

$$
d_{s}\left(\varepsilon_{a}, \varepsilon_{p}\right)=\inf \left\{h \in[0,1]: \varepsilon_{\operatorname{mia}\{o, b]}(x) \leqslant \varepsilon_{\max \left\{a h_{2}\right.}(x+h)+h, x \in[0,1 / h \mid\}\right. \text {. }
$$

The inequality on the right hand of (2.1) occurs in the following cases:

(a) $h \geqslant 1$.

(b) $\frac{1}{h} \leqslant \min \{a, b\}$, or equivalently $h \geqslant \frac{1}{\operatorname{mili} \mid a, b}$

(c) For every $x$ elmin $\{a, b], 1 / h \mid$ one has

$$
\begin{aligned}
& 1 \leqslant \varepsilon_{\max \{0.0 \mathrm{~b}}(x+h)+h \text { and } h<\frac{j}{\min |a \cdot b|} \\
& \Longleftrightarrow h<\min \left\{1, \frac{1}{\min (a, b)}\right\} \text { with } x+h>\max \{a, b\} \text { for all } x \in[\min \{a, b\}, 1 / h \mid \\
& \Leftrightarrow h<\min \left\{1, \frac{t}{\min \left\{a b_{l}\right.}\right\} \text { with } h \geqslant \max \{a, b\}-\min \{a, b\}=\{a-b
\end{aligned}
$$

for all $x \in] \min \{a, b\}, 1 / h[$.

Theorem 2.5. Let $\left(V, v, \tau, \tau^{*}\right)$ be a PN space where $V$ is a linear space and $v_{p}=\varepsilon_{\text {vpp }}$ and $\varphi=V \rightarrow R$ is a positive function such that for every $p, q \in V ; \varphi(q)-\varphi(p) \leqslant \varphi(q-p)$. Then $\left(V, r, \tau, \tau^{*}\right)$ is a semi-invariant PN space. 
Proof. It is enough we prove the below relation

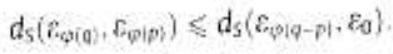

Let $p, q$ be in $V$. Then, from Lemma 2.4, one has:

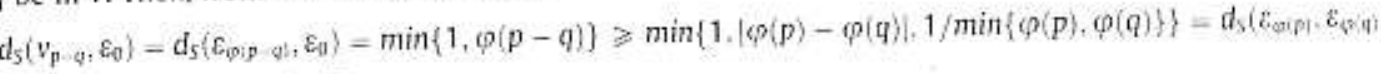

$$
\begin{aligned}
& =d_{5}\left(v_{p}-v_{q}\right) \text {. }
\end{aligned}
$$

Example 2.6. The quadruple $\left(V, v, \tau_{M}, \Pi_{M}\right)$ where $\left.V,\|\cdot\|\right)$ is a classical normed space and $v_{p}=\varepsilon_{Q i p}$, with $\varphi(p)=\frac{|p|}{1-|p|^{*}}$, is a semi-invariant $\mathrm{PN}$ space.

Proof. $\left(V, v, \tau_{M}, \mathbf{M}\right)$ is a PN space (see [6|) which is not a Šrstnev space. From Lemma $2.4 d_{s}\left(\varepsilon_{\mathrm{q}}(p-q), \varepsilon_{0}\right)=\frac{|p-q|}{1+|p-q|}$. On the other hand the function $\varphi(p)=\frac{|p|}{1+1+1}$ satisfies the condition given in Theorem 2.5

$$
\begin{aligned}
& \varphi(q)-\varphi(p)=\frac{\|q\|}{1+\|q\|}-\frac{\|p\|}{1+\|p\|} \\
& =\frac{\|q\|-\|p\|}{1+\|q\|+\|p\|+\|p\|\|q\|} \leqslant \frac{\|q\|-\|p\|}{1+\|q\|+\|p\|} \leqslant \frac{\|q-p\|}{1+\|q-p\|}-\varphi(q-p) .
\end{aligned}
$$

and the proof is complete.

It is possible to give sufficient conditions under which the inequality

$$
d_{s}\left(v_{p-q}, \varepsilon_{0}\right) \geqslant d_{s}\left(v_{p}, v_{4}\right)
$$

holds, as in the following theorem but we need before a lemma:

Lemma 2.7. According with some considerations in [9] one has:

$(i=1,2)$ be distance distribution functions in $\mathrm{A}^{*}$. Let $A_{\mathrm{i}}, i=1,2$ be the set defined vio

$$
A_{i}:=\left\{h \in[0,1]: \text { both }\left\{F_{i}, G_{i}, h\right\} \text { and }\left[G_{i}, F_{i}, h\right] \text { hoid }\right\} \text {. }
$$

Then,

$$
\begin{array}{ll}
d_{5}\left(F_{1}, G_{1}\right)<d_{s}\left(F_{2}, G_{2}\right) & \text { if and only if } A_{1} \supset A_{2} \\
d_{s}\left(F_{1}, G_{1}\right)=d_{5}\left(F_{2}, G_{2}\right) & \text { if and only if } A_{t}=A_{2} .
\end{array}
$$

Proof. Let us recall that $d_{s}\left(F_{i}, G_{i}\right):=\inf A_{i,}(i=1.2)$. By the definition of $A_{i}$, if $h_{0} \in A_{i}$, then $\left.\left.h_{0}, 1\right] \subset A_{i} \subset j 0,1\right]$; from Lemma 1.1 , if $h=\inf A_{1}>0$, then

$$
\left[F_{1}, G_{;}, h\right] \text { and }\left[G_{1}, F_{1}: h\right] \text {. }
$$

hold.

Moreover

$$
A_{i}= \begin{cases}0,1] & \text { if } d_{s}\left(F_{i}, G_{i}\right)=0 \\ {\left[a_{i}, 1\right]} & \text { if } d_{5}\left(F_{i}, G_{i}\right)=a_{i} .\end{cases}
$$

Theorem 2.8. Let $C$ be an associative copula and $\left(V, v, \tau_{C}, \tau^{+}\right)$a ${ }^{\prime} N$ space. Then $\left(V, v_{C}, \tau^{\prime}\right)$ is semi-invariant.

Proof. Since $C$ is an associative copula, then $C$ is a continuous $\left[-\right.$-norm and as a consequence one can say that $\tau_{c}$ is a continuous triangle function.

When calculating $d_{s}\left(v_{p}, \varepsilon_{0}\right)$ notice that the condition $\left[\varepsilon_{0}, v_{p \rightarrow q} ; i\right]$ is always right. The other one, $\left[v_{p-q}, \varepsilon_{0} ; i t\right.$, tell us that given $h \in 0,11$ and for all $x \in 0,1 / h$, one has the relation $1 \leqslant v_{p-q}(x+h)+h$ holds. Following Lemma 2.7 assume that $F_{1}=v_{p}$. $G_{1}=v_{Q}$ and $F_{2}=v_{p-q} . G_{2}=\varepsilon_{0}$. We have to prove that given $h \in[0,1]$ and for all $x \in[0,1 / h \mid$, if

$$
1-h \leqslant v_{p-q}(x+h) \text {. }
$$

then

$$
v_{p}(x) \leqslant v_{v}(x+h)+h \text { and } v_{4}(x) \leqslant v_{D}(x+h)+h .
$$

hold. We only prove the second inequality because the other one is symmetrical: it suffices to interchange $p, q$.

For every $x \in \mid 0,1 / h$ and applying (2.3) one has 


$$
\begin{aligned}
v_{p}(x+h)+h & \geqslant \tau_{C}\left(v_{p-q}, v_{\tau}\right)(x+h)+h=\sup \left\{C\left(v_{p-q}(u), v_{q}(v)\right): u+v=x+h\right\}+h \geqslant \sup \left\{C \left(v_{p-u}\left(u_{i}, v_{4}\{v)\right): u+v\right.\right. \\
& =x+h, v(x, u) h\}+h \geqslant \sup \left\{C\left(1-h, v_{q}(v)\right): v<x\right\}+h=C\left(1-h, v_{q}(x)\right\}+h .
\end{aligned}
$$

Moreover, since $\mathrm{C}$ is a copula, it follows from (1.2) that

$$
C(1,1)-C\left(1, v_{9}(x)\right)-C(1-h, 1)+C\left(1-h, v_{4}(x)\right)=1-v_{9}(x)-(1-h)+C\left(1-h, v_{4}(x)\right) \geqslant 0 .
$$

so that

$$
C\left(1-h, v_{q}(x)\right)+h \geqslant v_{q}(x),
$$

As a consequence of this theorem, if $\left\langle V, v, \tau_{\gamma}, \tau^{\cdot}\right)$ is a PN space such that $\tau_{f} \geqslant \tau_{c}$. with $C$ an associative copula, then $\left(V, V, \tau_{T}, \tau^{*}\right)$ is semi-invariant.

\section{Corollary 2.9 .}

(a) Every equilateral space $\left(V, F, n_{M}\right)$ is semi-invariant.

(b) Every simple PN space $(\mathrm{V}, H-H, G, M)$ is semi-imvariant.

(c) Every EN space ( $S, v$ ) is semi-invariant.

Before introducing a new class of PN spaces we need the following technical lemma from [7].

Lemma 2.10. Let $f:|0,+\infty| \rightarrow \mid 0,1]$ be a right-continuous non-increasing function. Let we define $f .1](1):=0$ and $f^{-1}(y):=\sup \{x: f(x)>y\}$ for all $y \in[0,1]\left\langle\left(f^{-1} \mid(y)\right.\right.$ might be infinite). If $x_{0} \in|0,+\infty|$ and $y_{0} \in[0,1]$, then the following facts are equivalent: $(a) f\left(x_{0}\right)>y_{0}:(b) x_{0}<f^{-1}\left(y_{0}\right)$.

Proof. If $f\left(x_{0}\right)>y_{0}$ then $f^{-1}\left(y_{0}\right)=\sup \left\{x: f(x)>y_{0}\right\} \geqslant x_{0}$. If we suppose that $\sup \left\{x: f(x)>y_{0}\right\}=x_{0}$. then $f(x) \leqslant y_{0}$ for every $x>x_{0}$. Thus $f\left(x_{0}\right)=f\left(x_{0}+\right) \leqslant y_{0}$, against the assumption; whence $(a) \Rightarrow$ (b). The converse result is an immediate consequence of the monotonicity of $f$.

The following theorem (see [7]) introduces a new class of PN spaces - which generalizes an example in [9|- and also provides some properties of the spaces in that class. As has been said above, such properties are interesting in order for the purposes of this paper.

Theorem 2.11. Let $(V . \|-1)$ a normed space and let $T$ be a continuous $t-n o r m$. Let $f$ be a function as in lemma 2.9, and satisfying the following two properties:

(a) $f(x)=1$ if and only if $x=0$;

(b) $f(\|p+q\|) \geqslant T(f(\|p\|\rangle . f(\|q\|))$ for every $p, q \in V$.

$$
\begin{aligned}
& \text { If } v: v \rightarrow \Delta^{+} \text {is given by } \\
& v_{p}(x)= \begin{cases}0, & x \leqslant 0 . \\
f(\| p \mid), & x \in 0,+\infty[, \\
1, & x=+\infty,\end{cases}
\end{aligned}
$$

for every $\mathrm{p} \in \mathrm{V}$, then $\left(V, V, \tau_{T}, \tau_{M}\right)$ is a Menger $P N$ space satisfying the following properties:

(Fv) $\left(V, v, \tau_{1}, \tau_{M}\right)$ is a TV space:

(F2) $\left(V, v, \tau_{T}, \tau_{M}\right)$ is normable:

(F3) If $p \in V$ and $t>0$, then the strong neighborhood $N_{p}(t)$ in $\left(V, v, \tau_{T}, \tau_{H}\right)$ is not $g$-bounded, but $N_{p}(t)$ is topologically bounded whenever $N_{p}(t) \neq V$;

(F4) $\left(V, v, \tau_{T}, \tau_{M}\right)$ is not a Serstev space:

(F5) $\left(V, v, \tau_{T}, \tau_{M}\right)$ is not a characteristic PN space.

Now we consider some special cases and use the preceding theorem in order to give some examples.

Example 2.12. Suppose that, in Theorem $2.11, T=\Pi$. Then, property (b) reads $f(\|p+q\|) \geqslant f(\|p\|) f(\|q\|)$ for all $p . q \in V$. It is not difficult to prove that, under the given assumptions on $f$, property $(b)$ is equivalent to the following one:

$$
f(x+y) \geqslant f(x) f(y), \quad \text { for all } x, y \in|0, \infty| \text {. }
$$

The following are examples of functions $f$ satistying the assumptions of Theorem 2.11: 


$$
\begin{aligned}
& f_{x, \beta}(x):=1-\frac{\beta}{\alpha}+\frac{\beta}{x+\alpha}, \quad 0 \leqslant \beta \leqslant \alpha, \\
& g_{z, \beta}(x):=1-\alpha+\alpha \exp \left(-x^{x}\right), \quad 0<\alpha \leqslant 1, \quad \beta>0 .
\end{aligned}
$$

Example 2.13. Take $T=W$ in Theorem. In this case property $(b)$ reads

$$
f(\|p+q\|) \geqslant f(\|p\|)+f(\|q\|)-1 \quad \text { for all } p, q \in V .
$$

Since $W$ is the smallest continuous $t$-norm, all the functions $f$ satisfying the assumptions of Theorem 2.11 with respect to any $l$-norm $T$ also satisfy such assumptions with respect to $W$. It is not hard to prove that. under those assumptions, property (b) is equivalent to the following one:

$$
1+f(x+y) \geqslant f(x)+f(y) \quad \text { for all } x, y \in] 0, \infty \text { : }
$$

For instance, the following functions satisfy this property but not that considered in Example 2.12, since they do not satisfy (2.5);

$$
h_{x, \beta}(x):= \begin{cases}1-\alpha x, & 0 \leqslant x \leqslant \beta . \\ 1-\alpha \beta, & x>\beta .\end{cases}
$$

Theorem 2.14. Every PN space belonging to the class considered in 7heorem 2.11 is semi-invariant.

Proof. Since $d_{s}\left(v_{p-q}, \varepsilon_{0}\right)=\inf \left\{h \in\left[0,1\left[: v_{p-q}\left(h^{\prime}\right)>1-h\right\}\right.\right.$ one has $f(\|p-q\|)>1-h \Rightarrow h>1-J(\|p-q\|)$, and it follows:

$$
d_{5}\left(v_{p-4}, \varepsilon_{0}\right)=1-f(\|p-q\|) \text {. }
$$

By the other hand, according with (1.1).

$$
d_{s}\left(v_{p}, v_{q}\right)=\inf \{h \in] 0,1\left[\text { both }\left[v_{u}, v_{p}: h\right] \text { and }\left[v_{p}, v_{q} ; h \mid h o l d\right\}\right. \text {. }
$$

Suppose, without lost of generality, $\mid p\|\geqslant\| q \|$, then $\left[v_{q}, v_{p}: h\right]$ is equivalent to $f(\|p\|) \leqslant f(\|q\|\rangle+h$. In fact this inequality is strict. Moreover, from $\left[v_{p}, v_{q}: h \mid\right.$ one has $h_{1} \geqslant f(\|q\|)-f(\|p\|)$, hence

$$
d_{5}\left(v_{p}, v_{\eta}\right)=f(\|q\|\rangle-f(\|p\|) \text {. }
$$

Now we need to investigate under which particular conditions one has for the PN spaces considered in Theorem 2.11 the inequality

$$
1-f(\|p-q\|) \geqslant f(\|q\|)-f(\|p\|\rangle \text {. }
$$

If one chooses $f$ among the type's functions $f_{x, \beta}$, then it is not difficult to check that

$$
\frac{\|p\|-\|q\|}{\|p\|\|q\|+(\|p\|+\|q\|) \alpha+x^{2}} \leqslant \frac{\|p-q\|}{\alpha^{2}+\|p-q\| x} .
$$

and the inequality $d_{s}\left(v_{p-q}, v_{0}\right) \geqslant d_{s}\left(v_{p}, v_{q}\right)$ holds. And if one chooses $f$ among the type's functions $g_{x \beta}$, then the PN spaces of Theorem 2.11 are always semi-invariant strictly: since

$$
d_{5}\left(v_{p-q}: \varepsilon_{0}\right)=1-g_{2 .}(\|p-q\|)=\alpha-\alpha^{-1 p-4 f^{\prime}},
$$

and

$$
d_{5}\left(v_{p}, v_{q}\right)=g_{x, \beta}(|q|)-g_{2, s}(\|p\|)=\alpha e^{-i 4^{p}}-\alpha e^{-\mid j \|^{4}},
$$

one has only to check the inequality $1-e^{-\left\{p-\left.q\right|^{3}-1\right.} \geqslant e^{-4 q}\left(1-e^{-\left(\left.|p|\right|^{r}-\left.4 q\right|^{5}\right)}\right)$, which is equivalent to check on the inequality $1-e^{-\left\{P-\left.q\right|^{p}\right.} \geqslant 1-e^{-\left(\mid P P^{2}-1 q^{2}\right.}$, and this is true because of the well known inequality $(1-s)^{p} \geqslant 1-s^{p}$. In fact, the inequality is strict and the verification is complete. Finally, if one chooses $f$ among the type's functions $h_{\alpha, f}$ the PN spaces considered in Theorem 2.11 are also semi-invariant.

Definition 2.15 [14]. A topological space is called normal space, if any two disjoint closed subset of it can be separated by open sets.

Theorem 2.16. Every semi-invariant PN space $\left(V, v, \tau, \tau^{*}\right)$ is normal.

Proof. If $A, B$ are closed subset of $V$ we should construct two disjoint open subset $U, W$ of $V$ such that $A \subset U$ and $B \subset W$.

Let $U:=\left\{p \in V: \inf _{e \in A} d_{s}\left(v_{y-a}, s_{0}\right)<\inf _{b=0} d_{s}\left(v_{\pi-b}, \varepsilon_{0}\right)\right\}$. If $p \in A$ then $\inf _{6 \in A} d_{s}\left(V_{p-c}, \varepsilon_{0}\right)=0$ and hence $A \subset U$. Now we prove that $U$ is open.

Suppose $p \in U, \alpha_{p}=\inf d_{s}\left(v_{p-q}, \varepsilon_{0}\right)$ and $\beta_{p}=\inf d_{s}\left(v_{p-b}, \varepsilon_{0}\right)$. Therefore $x_{p}<\beta_{p}$.

For every $\epsilon>0$ there exist $a_{0} \in A$ such that $d_{s}\left(v_{p-n_{0}}, c_{0}\right)<x_{p}+\epsilon$, 
For every $p \in U$ we will show that $N_{p}\left(\frac{\theta_{p}-\alpha_{p}}{2}\right) \subset U$. Let $Q \in N_{p}\left(\frac{\theta_{p}-x_{2}}{2}\right)$ then $d_{S}\left(v_{q_{i}-p}, \varepsilon_{0}\right)<\frac{\theta_{p}-\alpha_{p}}{2}$ and

$$
\inf d_{s}\left(v_{q-a}, \varepsilon_{0}\right) \leqslant \inf d_{S}\left(v_{q-a}, v_{q-p}\right)+d_{S}\left(v_{4-p}, \varepsilon_{0}\right) \leqslant \inf d_{S}\left\langle v_{q-11}, v_{0-p}\right\rangle+\frac{\beta_{p}-\alpha_{p}}{2} .
$$

The space $\left(V, v, \tau, \tau^{*}\right)$ is a semi-invariant and hence

$$
\text { inf } d_{S}\left(v_{q-a}, v_{q-n}\right) \leqslant \inf d_{S}\left(v_{p-n}, c_{0}\right)=\alpha_{F} .
$$

Therefore $d_{s}\left(v_{q-q}+\varepsilon_{0}\right) \leqslant \alpha_{p}+\frac{\theta_{p}-x_{p}}{2}=\frac{\theta_{p}+x_{p}}{2}<\beta_{p}$ and $q \in U$.

Similarly if $W:=\left\{p \in V:\right.$ inf $d_{s}\left(v_{p \sim a}, f_{0}\right)>$ inf $\left.d_{s}\left(v_{p-b}, \vec{L}_{0}\right)\right\}$ then $W$ is open subset of $V$ such that $B \subset W$ and the construction of $U$ and $W$ shows that $U \cap W=0$.

Immediately the next corollaries come from Theorem 2.8 .

Corollary 2.17. Urysohn's lemma for PN spaceAny two disjoint subset of every semi-invariant PN space can be separated by a contimuous function.

Corollary 2.18 (The Tietze extension Thearem for PN space). If $A$ is any closed subset of the semi-invariant PN space (V.v. $\left.\tau, \tau^{*}\right)$ and $f \in C(A,[a, b])$ then there exists $F \in C(V,|a, b|\}$ such that $F \mid A=f$.

Corollary 2.19. If $A$ is any closed subset of the semi-invarian $P N$ space $\left(V, v, \tau, \tau^{*}\right)$ and $f \in C(A)$ then there exists $F \in C(V)$ such that $F \mid A=f$.

Open problems, One needs several previous results.

Definition $2.20[11]$. Let $Z^{-}$be the set of all binary operations, $L$, on $R^{-}$and $R a n(L)=R^{-}$satisfying the following conditions:

(i) $L$ is non-decreasing in each place,

(ii) $L$ is continuous on $\mathrm{R}^{+} \times \mathrm{P}^{+}$except at the most in $(0, \infty)$ and $(\infty, 0)$.

Theorem $2.21[11]$. Let $T$ be a left-continuous $t$-norm and let $L \in \mathscr{Z}$ satisfying the following conditions:
(i) $l$ is commutative,
(ii) $L$ is associntive.
(iii) $u_{1}<u_{2}$ and $v_{1}<v_{2}$ imply $L\left(u_{1}, v_{1}\right)<L\left(t_{2}, v_{2}\right)$.
(iv) $L(x, 0)=x$, then the funcrion $\tau_{\Gamma \perp}$ is a triangle function.

The condition ( $i v$ ) of the Theorem 2.21 implies that $L \geqslant M a x$, and then $\tau_{T, L} \leqslant \prod_{T}$.

Corollary 2.22. Let $L \in S$ satisfying the hypothesis of Theorem $2.2 \mathrm{I}$. Then the following statements hoid

(i) $L \geqslant$ Max (trivial and known)

(ii) (see [9]) $L=\operatorname{Max} \Longleftarrow L\langle a, a\rangle=a$ for every $a \in(0, \infty)$.

Theorem 2.23. Let $T$ be an Archimedean t-norm and assume that $L \in \mathscr{Z}$ satisfies tite hypothesis of the Theorem 2.21 . Then the following statements are equivalent:

(i) $\tau_{T, L}$ is a triangle function no having non-trivial idempotents in $\Delta^{\prime}$.

(ii) $L$ satisfies the condition $L(x, x)>x$ for every $x \in(0, \infty)$.

Example 2.24. This theorem provides us with many examples of Archimedean triangle functions: it suffices to have $L=K_{x}(x, y)=\left(x^{x}+y^{2}\right)^{1 / x}$ defined for every $x, y \in \mathbb{R}^{-}$and for $x \geqslant 1$.

Let us recall the definition of $\tau_{t, K_{z}}$ :

$$
\tau_{T K_{3}}=\sup \left\{T\langle F(u), G(v)): u^{x}+v^{x}=x^{x}\right\}
$$

Applying the previous example, and by Theorem 7.2 .12 in |11|, one has: $K_{2}<K_{1}$ and $T_{1} \leqslant T_{2}$ implies that $\tau_{\gamma_{1}, K_{1}} \leqslant \tau_{T_{2}, K_{2}}$. In analogous way, as $K_{4} \leqslant K_{2}$, the inequality $\tau_{T_{1}, K_{2}} \leqslant \tau_{T_{2}} X_{4}$.

We are ready to propose the following open problems all of which need from the computation sciences. 
(1) Is any $\alpha$-simple space invariant or semi-invariant? If not, are there any conditions under which it is? Also, if not, why not?

(2) In the following examples and for the moment, we do not know the answer to the same questions mentioned in (1).

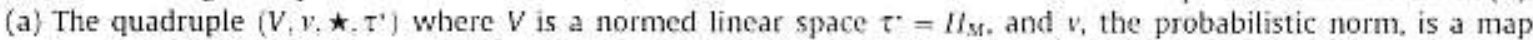
$r: V \rightarrow \Delta^{+}$defined via $v_{p}(x):=\frac{\gamma}{x+1} e^{-|v|}$, and $\star$ the triangle function convolution, is a PN space; it is neither a Serstnev space nor a topological vector space.

(b) The quadruple $\left\langle V, v, \Pi_{\pi}, \Pi_{M}\right)$ where $V$ is a normed linear space and $v$, the probabilistic norm, is a map $v: V \rightarrow A^{+}$ defined via $v_{p}\langle x\rangle:=e^{p}$, is a PN space and a topological vector (in short, TV) space,

(c) Let $\left(V, v, \tau, \tau^{*}\right)$ a PN space and let $\tau^{*}$ be non-Archimedean triangle function, Let $f, g$ be functions satisfying the following conditions:

(i) $f$ is a continuous non-increasing from $[0,+\infty]$ into $[0,1]$ and $f(0)=1$,

(ii) $g$ is a function from $\mathbb{R}^{\prime} \times \mathbb{R}^{\prime}$ into $[0,1]$, continuous in the first and in the second places, non-decteasing in the first place and non-increasing in the second place with $g(x, 0)=1$. Then the quadruple $\left(V, v, \tau, \tau^{*}\right)$ where the probabilistic norm $v$ is defined via

$v_{p}(x)=f(\|p\|) \cdot g(x, \| p||)$.

is a topological vector space.

(d) With the same assumptions of the previous example one has that the quadruple $\left(V, \gamma, \Pi_{\eta}, \Pi_{m}\right)$ where

$$
v_{p}(x)=\frac{1}{1+\|p\|} \cdot \frac{x}{x+\|p\|}
$$

is a PN space that is a TV space and it is not a PN space of Šerstnev. $\left(V, v, \Pi_{i}, \Pi_{\mathrm{M}}\right)$ is not a strict PN space, and it is normable.

\section{Application in physics}

Menger sponge is a random space which could be used for instance to predict the Background microwave radiation (see El Naschie and also He's Book [4]).

\section{Conclusions}

In this work, we have analyzed some detail of semi-invariance for some class of PN spaces. We have shown that PN spaces are normal spaces. A detailed study of how we can have the Urysoln's Lemma and Tietze extension Theorem for PN spaces is given.

\section{Acknowledgement}

The Second author was supported by grants from Ministerio de Ciencia y Tecnologa (MTM2006-12218) of Spain.

\section{References}

[1] Alsina C. 5chweizer B. Sklar A. On the definition of a probabilistic normed space, Aequationes Math 1993;46:91-8.

2) El Naschie MS. On the uncertainty of Cantorian reometry and the two slit experiment. Chaos. Solitons \& Fractals 1998:9:517-29.

[3] El Naschie MS. On the unification heterotic strings theory and $2^{\text {* }}$ theory. Chalos. Solitons \& Fractals 2000:11:397-407.

[4] Ji-Huan He, El Naschie MS, Transfinite plyssus. Beijng; China Education and Culture publishing Co:; 2006.

[5] Lafuerza-Cuiltén B, Rodriguez Lallena JA. Sempi C. Some elasses of probabilistic normed spates. Rend Mat 1997:17:237-52,

[6] Lafuerza-Guillén B. Latest results on probabilistic normed spaces. Int J Math Anal 2006;3:269-309.

[7] Lafuerza-Cuillén B. Redrózez Lallena JA, Sempi C. Normability of probabilistic normed spaces. Note di Matemarica, in press.

[8] Lafuezza-Guillén B, Rodriguez Lallena JA. Sempi C. A study of boundedness in probabalistic normed spates. J Math Anal Appl 1999:232:183-96.

19] Lafuerza-Guillén B. Primeros resultados en el estudio de los espacios normados probabilísticos con nuevos concptos de acotación, Ph.D. Thesis, Universidad de Ameria, Spain: 1996.

|10| Saminger S. Sempi C. A primer on triangle funtrions I. Aequationes Math 2008;76:201-40.

111 Schweizer B, Sklar A. Probabilistic metric spsces. New York: Dover Publitation. Int. Mineola: 2005.

[12| Serstnev AN. On the motion of a random normet space. Dokl rkad Nauk SSSR 1963;149:280-3 JEnglish translation in Soviet Math Dokl 1963:4:388. 901.

[13] Sibley DA A metric for weak convergeruce of distribution functions. Rocky Mountain J Math 1971:1:127-30.

[14]. Wilansky A. Functional analysis. New York. Toronto, London: Blaisdell Publishing Company; 1964. 